\title{
Energies controlling nucleation and growth processes: The case of $\mathrm{Ag} / \mathrm{W}(110)$
}

\author{
Jones, G. W.; Marcano, J. M.; Nørskov, Jens Kehlet; Venables, J. A.
}

Published in:

Physical Review Letters

Link to article, DOI:

10.1103/PhysRevLett.65.3317

Publication date:

1990

Document Version

Publisher's PDF, also known as Version of record

Link back to DTU Orbit

Citation (APA):

Jones, G. W., Marcano, J. M., Nørskov, J. K., \& Venables, J. A. (1990). Energies controlling nucleation and growth processes: The case of Ag/W(110). Physical Review Letters, 65(26), 3317-3320.

https://doi.org/10.1103/PhysRevLett.65.3317

\section{General rights}

Copyright and moral rights for the publications made accessible in the public portal are retained by the authors and/or other copyright owners and it is a condition of accessing publications that users recognise and abide by the legal requirements associated with these rights.

- Users may download and print one copy of any publication from the public portal for the purpose of private study or research.

- You may not further distribute the material or use it for any profit-making activity or commercial gain

- You may freely distribute the URL identifying the publication in the public portal 


\title{
Energies Controlling Nucleation and Growth Processes: The Case of Ag/W(110)
}

\author{
G. W. Jones, ${ }^{(1)}$ J. M. Marcano, (1) J. K. Nørskov, ${ }^{(2)}$ and J. A. Venables ${ }^{(1),(a)}$ \\ (1) School of Mathematical and Physical Sciences, University of Sussex, Brighton BN1 9QH, England \\ ${ }^{(2)}$ Laboratory of Applied Physics, Technical University of Denmark, Building 307, DK-2800 Lyngby, Denmark \\ (Received 30 July 1990)
}

\begin{abstract}
The nucleation density $N_{x}(T)$ of $\mathrm{Ag}$ islands on the 2-monolayer-thick silver intermediate layer on $\mathrm{W}(110)$ is measured by UHV scanning electron microscopy and compared with kinetic nucleation equations. This yields a well-determined value of $E_{d}+2 E_{b}=0.65 \pm 0.03 \mathrm{eV}$, with pair-binding energy $E_{b}=0.25 \pm 0.05$, diffusion energy $E_{d}=0.15 \mp 0.10$, and adsorption energy $E_{a}=2.2 \pm 0.1 \mathrm{eV}$. Values for $\mathrm{Ag} / \mathrm{Ag}(111)$ are calculated in effective-medium theory, giving $E_{a}=2.23, E_{b}=0.28, E_{d}=0.12$, and $E_{d}+2 E_{b}=0.68 \mathrm{eV}$. Observations on vicinal and impure surfaces are reported, and implications for defect binding energies discussed.
\end{abstract}

PACS numbers: $68.55 .-\mathrm{a}$

The understanding of epitaxial crystal growth in atomistic terms has been a long-standing goal for the physics of materials. In recent years, much experimental progress has been made via the combined use of surface science and (electron) microscope techniques. ' In met$\mathrm{al} /$ metal systems, the Stranski-Krastanov (or layer-plusisland) growth mode has been found to be the norm rather than the exception. For $\mathrm{Ag} / \mathrm{W}(110)$ (Refs. 2-4) an intermediate layer 2 monolayers (ML) thick is formed first, and then $\mathrm{Ag}$ islands grow in the (111) orientation. The crystallography of the first two layers is very similar to $\mathrm{Ag}(111)$, but is slightly distorted to match the substrate better.

The nucleation and growth of islands on top of this intermediate layer has been modeled using kinetic rate equations. ${ }^{5}$ The advantage over Monte Carlo methods is that we can cope with the large range of length and time scales involved in these diffusion and clustering processes. Applied to measurements of the nucleation density $N_{x}$ as a function of deposition rate $R$ and substrate temperature $T$, our approach has yielded a three-parameter fit in terms of the surface diffusion energy $E_{d}$, the pairbinding energy $E_{b}$, and, at the highest temperatures, the adsorption energy $E_{a}$. These parameters are just those that can be most readily calculated by a priori theory. The development of theories of metals based on the local-density approximation means that calculations involving large numbers of atoms can be attempted, including relaxation of atomic positions. In particular, the "effective-medium approach" has shown itself capable of estimating vacancy and surface energies and related quantities with reasonable precision. Several examples are given in Refs. 6 and 7.

In this Letter we present an extensive new data set, $N_{x}(T)$, for $\mathrm{Ag} / \mathrm{W}(110)$, and deduce the diffusion, binding, and adsorption energies by comparison with rate equations. These parameters are then calculated by the effective-medium theory, and are shown to be in remarkable agreement. In addition, we present data on vicinal surfaces, and surfaces contaminated with adsorbed $\mathrm{CO}$. By comparing $N_{x}(T)$ on such surfaces with the rate- equation predictions, we are able to deduce an upper limit to the binding energy to these special sites, and to comment on the nature of the bonding involved.

The experiments were conducted in the UHV scanning electron microscope at the University of Sussex, using techniques described previously. Thin islands were observed with high contrast using biased secondary electron imaging. ${ }^{8}$ In addition to measurements of nucleation density, we also deposited $\mathrm{Ag}$ through a mask of holes, in order to observe surface diffusion directly. ${ }^{9}$ Two examples of 5-monolayer $(\mathrm{ML})$ deposits at 300 and $400^{\circ} \mathrm{C}$ on a tungsten sample whose orientation is very close to (110) are shown in Figs. 1(a) and 1(b). The nucleation density $N_{x}$ in (a) is $1.5 \times 10^{7} \mathrm{~cm}^{-2}$, and the islands are $\sim 1 \mu \mathrm{m}$ across. The density in Fig. 1(b) is lower by a factor of around 5. Given that we have complete condensation at both temperatures, and that the intermediate layer is $2 \mathrm{ML}$ thick, the islands are about $30 \mathrm{ML}$, or $10 \mathrm{~nm}$, thick. It is clear that they grow much more readily laterally than vertically, a condition which is associated with defect-free islands. ${ }^{10}$ It is also known that the islands have their (111) planes accurately parallel to the substrate (110) plane, ${ }^{11}$ which we have checked in the present work by reflection high-energy electron diffraction.

For comparison, some islands grown on surfaces vicinal to (110) are shown in Figs. 1(c) and 1(d). The substrate misorientation can be gauged from the angle between the top face of the islands, which is (111), and the intersection of the front face with the substrate. In Fig. 1 (c), this angle is up to $5^{\circ}$; the corresponding step spacing on the substrate is of the order of $4 \mathrm{~nm}$ if the steps are single height and more if they are multiple. In some cases where multiple steps are clearly seen, such as Fig. 1 (d), the islands have a much larger height-to-width ratio, and they span the steps. This is almost certainly due to the growth on dislocation spirals generated at the steps. ${ }^{1,10}$ However, the intriguing point is that the nucleation density is not greatly enhanced in these situations, and that the adatoms move rather easily over the steps. This is in contrast to the behavior of adatoms on 


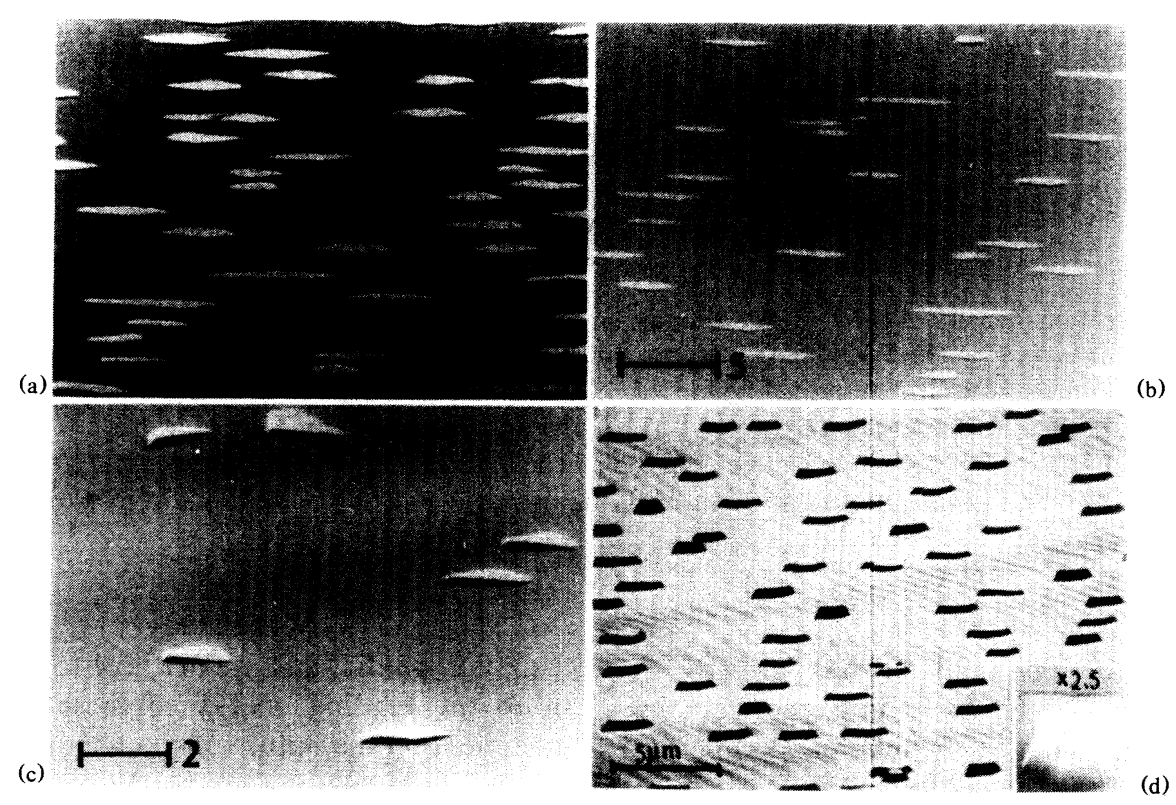

FIG. 1. Flat silver islands in (111) orientation on W(110); $5-\mathrm{ML}$ deposit at $R=0.3 \mathrm{ML} / \mathrm{min}$ at (a) $T=300^{\circ} \mathrm{C}$ and (b) $400^{\circ} \mathrm{C}$. (c) Islands on a vicinal surface in the same experiment as in (b). (d) Thicker islands spanning multiple steps on faceted vicinal surface (from Ref. 15). See text for discussion.

the bare substrate, which we have shown to be strongly influenced by the presence of steps, and by adsorbed $\mathrm{CO}$ impurities at the 0.1-ML level. Similar strong effects have been seen in related systems by scanning Auger microscopy of $\mathrm{Pd} / \mathrm{W}(110)$ (Ref. 12) and by low-energy and photoemission electron microscopy of $\mathrm{Cu} / \mathrm{Mo}(110) .{ }^{13}$

These observations tell us a lot about the bonding strengths between $\mathrm{Ag}$ and $\mathrm{W}(110)$ and between $\mathrm{Ag}$ and $\mathrm{Ag}$ (111). The nucleation-density data can be analyzed quantitatively to yield various energies indicative of these bonding strengths. In Fig. 2 we present the new $N_{x}(T)$ data, where we distinguish carefully between data on flat, clean surfaces and data on stepped or slightly contaminated surfaces. The data span the range $200<T$ $<500^{\circ} \mathrm{C}$. On the flat surfaces, the data agree with the lowest nucleation densities obtained previously, ${ }^{3}$ and in general are in very good agreement with those data. On the stepped or contaminated surfaces, the $N_{x}$ values are up to a factor of 2 higher.

Figure 2 also shows the fit with the rate-equation formulation for the two data sets. The best fit to the flat, clean substrate requires the energy combination $E_{d}$ $+2 E_{b}=0.65 \pm 0.03 \mathrm{eV}$, where the uncertainty corresponds to a factor-of- 2 variation in the preexponential frequency factors. ${ }^{5}$ This determination stems from the absolute value of $N_{x}(T)$, whereas the slope of the $\log \left(N_{x}\right)-1 / T$ plot determines $E_{d}$ and $E_{b}$ separately. The critical nucleus size is in the range 6-34 atoms over the $T$ range shown. By repeating the prediction with various $E_{d}$ and $E_{b}$ and calculating weighted rms deviations from the new data, we produced the contour plot of Fig. 3 from which we can estimate $E_{b}=0.23 \pm 0.05 \mathrm{eV}$ and
$E_{d}=0.18 \pm 0.10 \mathrm{eV}$. Given that similar plots for the previous data produced slightly higher $E_{b}$ and lower $E_{d}$ values, and the unreality of quoting errors better than $0.05 \mathrm{eV}$, our "best" estimate is $E_{b}=0.25 \pm 0.05$ and $E_{d}=0.15 \pm 0.10 \mathrm{eV}$, where the errors are somewhat con-

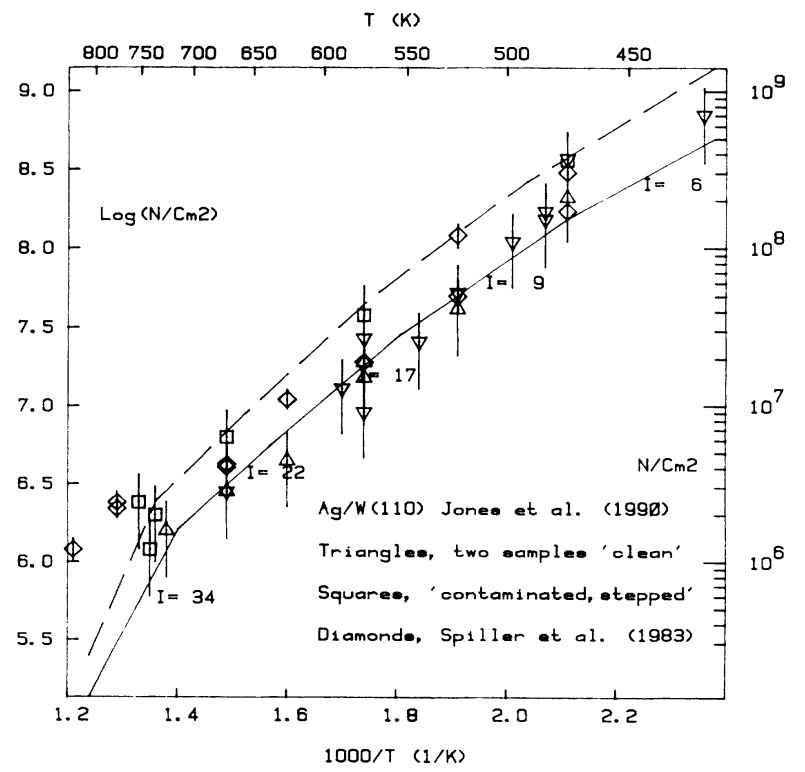

FIG. 2. Nucleation density of silver islands on $\mathrm{W}(110)$ as a function of $T^{-1}$. Full line: $E_{a}=2.1, E_{b}=0.25, E_{d}=0.135 \mathrm{eV}$, compared to data on the flattest, cleanest samples; dashed line: $E_{d}=0.185 \mathrm{eV}$, other parameters unchanged, compared to data on stepped and/or slightly contaminated samples. Deposition rate $R=0.3 \mathrm{ML} / \mathrm{min}$. 


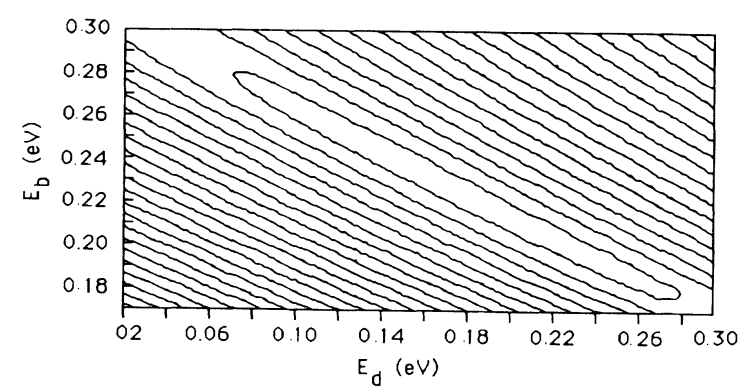

FIG. 3. Goodness-of-fit plot based on new clean-substrate data presented here. The contours represent the root-meansquare deviation of the prediction from the experimental points on a $\log _{10}$ scale; the minimum (closed) contour is 0.15 , increasing in units of 0.1 . See text for discussion.

servative. The adsorption energy only influences the fits at the highest temperatures; we can say that $E_{a}$ is greater than $2.1 \mathrm{eV}$, as shown in Fig. 2, with a preferred value around $2.2 \mathrm{eV}$. If the binding energy in small $\mathrm{Ag}$ clusters is similar to that in bulk $\mathrm{Ag}$, we would have $E_{a}+3 E_{b}=L$, where $L=2.95 \mathrm{eV}$ is the sublimation energy. On this basis $E_{a}=2.95 \pm 0.01-3(0.25 \pm 0.05)$ $=2.20 \pm 0.18 \mathrm{eV}$. These estimates are clearly close to each other.

The above quantities have been calculated within the effective-medium theory, which is an approximate method for calculating total energies. ${ }^{6}$ The total-energy expression is derived from the density-functional theory and includes explicitly the many-body nature of the interatomic interactions in a metallic system. It has been shown to give good results for surface energies, for relaxations of interlayer distances at surfaces, and for surface reconstructions. ${ }^{6,7}$ In the present application the parameters entering the theory have been taken from the cohesive and elastic properties of bulk $\mathrm{Ag}$ as specified in Refs. 7 and 14.

The calculations have been performed for $\mathrm{Ag}$ on a semi-infinite $\mathrm{Ag}(111)$ surface rather than $2 \mathrm{ML}$ of $\mathrm{Ag}(111)$ on $\mathrm{W}(110)$. Since the lattice constants are very similar, this should give rise to only small errors. For each configuration the total energy was minimized with respect to the coordinates of the $\mathrm{Ag}$ atoms. A large $(4 \times 4)$ surface unit cell with one, two, or three adatoms was used in order to minimize interactions between adatoms in adjacent cells. The resulting interaction energies are shown in Table I. Considering the approximate nature of the theory the agreement with the experimentally deduced numbers is remarkable.

Both the experimental and the calculated numbers show a pronounced nonlinearity in the binding energy of the $\mathrm{Ag}$ adatoms with coordination number, or number of nearest-neighbor bonds. A single adatom on the surface thus has only three "bonds" and a total binding energy of $2.23 \mathrm{eV}(0.74 \mathrm{eV}$ per bond) whereas the extra bond between two adatoms on the surface only holds $0.29 \mathrm{eV}$. This is a direct consequence of the many-body nature of
TABLE I. The adsorption energy $\left(E_{a}\right)$, diffusion energy $\left(E_{d}\right)$, and pair-binding energy $\left(E_{b}\right)$ for a dimer and a trimer of $\mathrm{Ag}$ on an $\mathrm{Ag}(111)$ surface calculated using the effectivemedium theory.

\begin{tabular}{llllc}
\hline \hline & $\begin{array}{c}E_{a} \\
(\mathrm{eV})\end{array}$ & $\begin{array}{c}E_{d} \\
(\mathrm{eV})\end{array}$ & $\begin{array}{c}\text { Dimer } \\
E_{b}(2) \\
(\mathrm{eV})\end{array}$ & $\begin{array}{c}\text { Trimer } \\
E_{b}(3) \\
(\mathrm{eV})\end{array}$ \\
\hline Theory & 2.23 & 0.12 & 0.29 & 0.27 \\
Experiment & $2.2 \pm 0.1$ & $0.15 \pm 0.10$ & $0.25 \pm 0.05$ \\
\hline \hline
\end{tabular}

the bonding. Relaxations of the interatomic distances only account for a small fraction (a few times $0.01 \mathrm{eV}$ ) of the binding energies. The nonlinearity of the interaction energy with coordination number is, however, not nearly as pronounced when more adatoms are added. The "bond energy" for a trimer of adatoms is thus close to that of the dimer. The average value, 0.28 , is quoted for $E_{b}$ as a result.

As a separate exercise, the observations on stepped and contaminated surfaces enable us to make some semiquantitative deductions, and to make contact with other work on the behavior of $\mathrm{Ag}$ in the first monolayer on W(110). ${ }^{4}$ The dashed line in Fig. 2 corresponds to a diffusion energy just $0.05 \mathrm{eV}$ higher than the solid line appropriate to the clean surface. At $T=600 \mathrm{~K}$ this corresponds to a diffusion coefficient that is lower by a factor of 2.6 than that for the clean surface, assuming that binding energies have not been affected. If the adatoms diffuse over the defective surface, and are bound to special sites by an extra binding energy $E_{s}$, and the concentration of such sites is $N_{s}$, then we would expect that $D$ is reduced by a factor $1+\left(N_{s} / N_{0}\right) \exp \left(E_{s} / k T\right)$, where $N_{0}$ is the monolayer density. Thus if $N_{s} / N_{0}$ is around 0.1 , corresponding to an impurity concentration of this order or a vicinal angle of around $5^{\circ}$, the factor of 2.6 at 600 $\mathrm{K}$ requires $E_{s}$ to be not more than $2.8 k T$, or $0.15 \mathrm{eV}$. If we have a faceted surface such as Fig. 1(d), with the same adsorption energy on the facets as on (111) and the special site only at the corners, so that $N_{s} / N_{0}$ is reduced to 0.01 or 0.001 , this energy might rise as high as 0.26 or $0.38 \mathrm{eV}$. But this is unlikely, and the results almost certainly indicate that the special-site binding energy is less than around $0.2 \mathrm{eV}$.

This value is remarkably low, and suggests that the more obvious types of surface defects either grow out or become passivated in some way during growth. For example, a step $1 \mathrm{ML}$ high should bind an adatom by $2 E_{b}$, or a kink site by $3 E_{b}$, giving $E_{s}$ in the range $0.5-0.75 \mathrm{eV}$. Thus complete (111) layers will have a strong tendency to grow laterally, eliminating high-index facets. A step on the substrate surface has a height of $a_{0} / \sqrt{2}=2.23 \AA$; this is less than the 1-ML Ag height, $2.36 \AA$, but not by much. Maybe such a step will induce an inclined stacking fault or other defects to form, which have a small binding energy for adatoms; it certainly seems that pas- 
sivation of this type must be invoked if the low trapping energies on top of the 2-ML Ag layer are to be rationalized. Similar considerations apply to the observation that the surface can be contaminated with up to $0.1 \mathrm{ML}$ of adsorbed $\mathrm{CO}$ without a huge effect on the nucleation density. In this case, the $\mathrm{CO}$ molecule has a similar radius to, and probably a reduced interaction with, the other $\mathrm{Ag}$ atoms, so that the migrating $\mathrm{Ag}$ adatoms may even tend to avoid the sites on the surface which are contaminated, once the molecules have been buried by a layer of deposited silver.

The energies of $\mathrm{Ag}$ atoms in the first monolayer on W(110) have been obtained from the analysis of thermal-desorption and work-function data. ${ }^{2,4}$ The adsorption energy at low coverage is around $2.8 \mathrm{eV}$, rising to $3.55 \mathrm{eV}$ close to monolayer coverage. The difference corresponds to three $\mathrm{Ag}$ bonds of strength around 0.25 $\mathrm{eV}$ each, essentially the same as the value on the $\mathrm{Ag}$ intermediate layer. The difference in adsorption energies on the substrate and the 2-ML Ag layer $(2.8-2.2 \mathrm{eV})$, or in sublimation energies $(3.55-2.95 \mathrm{eV})$, both around $0.6 \mathrm{eV}$, indicate the greater strength of $\mathrm{Ag}-\mathrm{W}$ bonding. This value makes it qualitatively understandable that $\mathrm{Ag}$ diffusion on the bare $\mathbf{W}$ surface is considerably impeded by surface steps or by strongly chemisorbed molecules present in sub-0.1-ML quantities. Quantitative calculations of $\mathrm{Ag}$ on tungsten are, however, beyond the scope of the effective-medium theory at present.

The success of our experiment-theory comparison for elementary energies $E_{a}, E_{d}$, and $E_{b}$ on the clean stepfree $\mathrm{Ag}(111)$ surface suggests that similar calculations can usefully be attempted for small clusters up to the critical cluster size of a few tens of atoms, and for various step and defect configurations at surfaces which may be important in promoting nucleation or other surface reactions. The effective-medium approach should be especially effective, as demonstrated here, when we are comparing alternative configurations of the same number of atoms; then good accuracy can be expected with a highly simplified calculation which can be applied to the early stages of epitaxial growth in a variety of circumstances.

This work was supported by the Science and Engineer- ing Research Council and Central Electricity Research Laboratory (United Kingdom) and the Danish Research Council through the Center for Surface Reactivity. Some computations leading to Fig. 2 were done by J. G. Gonzalvez.

(a) Also at Department of Physics, Arizona State University, Tempe, AZ 85287.

'J. A. Venables, G. D. T. Spiller, and M. Hanbucken, Rep. Prog. Phys. 47, 399 (1984).

${ }^{2}$ E. Bauer, H. Poppa, G. Todd, and P. R. Davis, J. Appl. Phys. 48, 3773 (1977)

${ }^{3}$ G. D. T. Spiller, P. Akhter, and J. A. Venables, Surf. Sci. 131, 517 (1983).

${ }^{4}$ J. Kolaczkiewicz and E. Bauer, Surf. Sci. 155, 700 (1985); 175, 508 (1986).

${ }^{5}$ J. A. Venables, J. Vac. Sci. Technol. B 4, 870 (1986); Phys. Rev. B 36, 4153 (1987).

${ }^{6}$ K. W. Jacobsen, J. N. Nørskov, and M. J. Puska, Phys. Rev. B 35, 7423 (1987); K. W. Jacobsen, Comments Condens. Matter Phys. 14, 129 (1988).

${ }^{7}$ K. W. Jacobsen and J. K. Nørskov, in Structure of Surfaces II, edited by J. F. van der Veen and M. A. Van Hove, Springer Series in Surface Sciences Vol. 11 (Springer-Verlag, New York, 1988), p. 118

${ }^{8}$ M. Futamoto, M. Hanbucken, C. J. Harland, G. W. Jones, and J. A. Venables, Surf. Sci. 150, 430 (1985).

${ }^{9}$ G. W. Jones and J. A. Venables, Ultramicroscopy 18, 439 (1985)

${ }^{10} \mathrm{~J}$. W. Bermond and J. A. Venables, J. Cryst. Growth 64, 239 (1983).

${ }^{11}$ C. J. Harland, P. Akhter, and J. A. Venables, J. Phys. E 14, 175 (1981).

${ }^{12}$ R. Butz and H. Wagner, Surf. Sci. 87, 69 (1979); 87, 85 (1979).

${ }^{13}$ M. Mundschau, E. Bauer, and W. Swiech, Surf. Sci. 203, 412 (1988); E. Bauer, M. Mundschau, W. Swiech, and W. Telieps, Ultramicroscopy 31, 49 (1989).

${ }^{14}$ Using the nomenclature of Refs. 6 and 8, the parameters used are $E_{0}=2.95 \mathrm{eV}, E_{2}=0.86 \mathrm{eV}, E_{3}=0.40 \mathrm{eV}, \eta=2.49$ bohr $^{-1}, \eta_{1}=0.18 \mathrm{bohr}^{-1}, s_{0}=3.01$ bohrs, $n_{0}=0.0067 \mathrm{bohr}^{-3}$, and $\alpha n_{0}=16.73 \mathrm{eV}$.

${ }^{15}$ P. Akhter, J. Derrien, A. P. Janssen, and J. A. Venables, Inst. Phys. Conf. Ser. 52, 383 (1980). 


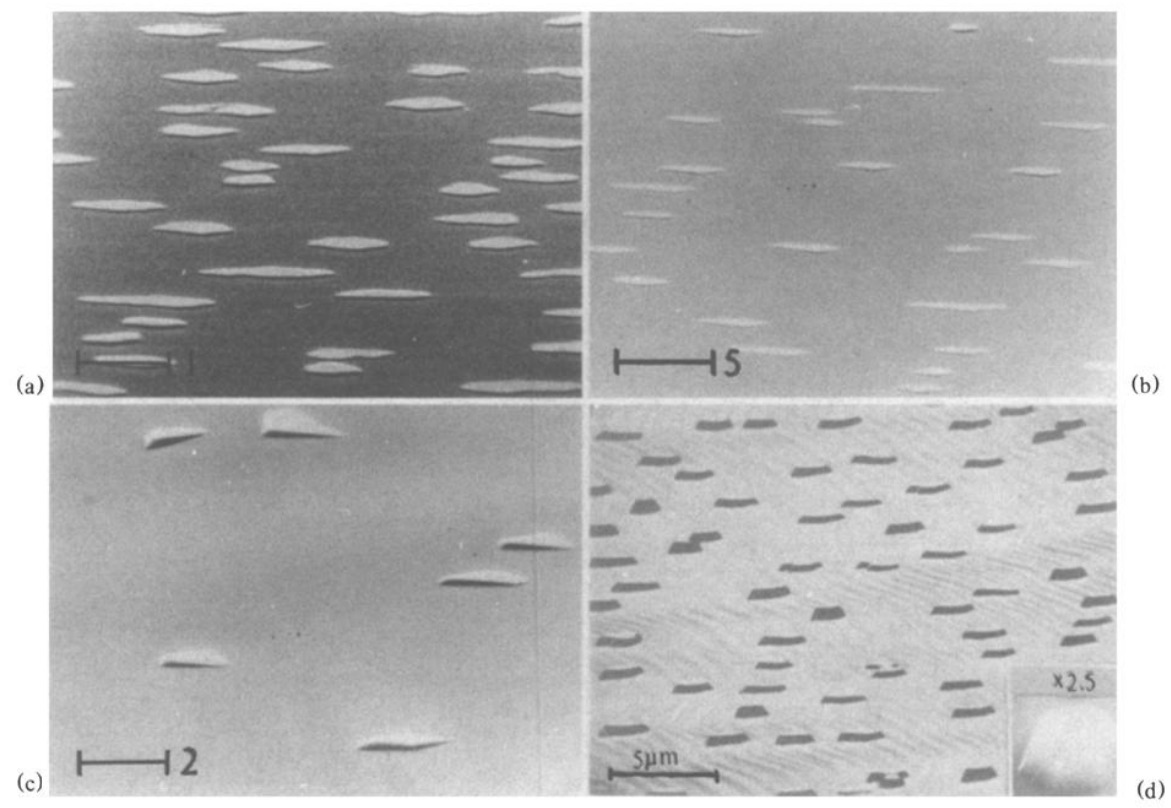

FIG. 1. Flat silver islands in (111) orientation on W(110); $5-\mathrm{ML}$ deposit at $R=0.3 \mathrm{ML} / \mathrm{min}$ at (a) $T=300^{\circ} \mathrm{C}$ and (b) $400^{\circ} \mathrm{C}$. (c) Islands on a vicinal surface in the same experiment as in (b). (d) Thicker islands spanning multiple steps on faceted vicinal surface (from Ref. 15). See text for discussion. 